Such radiations are frequently described by writers on the occult sciences as being emitted by the human body. For example, in the chapter on magnetism in M. de Dubor's recently published " Mysteries of Hypnosis," I read of a doctor who was making magnetic passes over a lady. "The subject was wearing a black dress, and the doctor had his back to the light. Suddenly, in the semi-darkness which surrounded him, he observed a greyish vapour, like the fumes of a cigarette, issuing from the tips of his fingers, and, with especial clearness, from the index and the middle fingers. Moreover, the index fingers of the two hands seemed to be united by a luminous arc or semicircle. . . . Other persons, on the doctor's invitation, drew near and observed the same phenomenon. .. . Then the room was darkened. . . . In the darkness, twelve of the witnesses perceived nothing at all, and the remaining six perceived only very little."

M. de Dubor and the whole occult school explain the glow, or aura, seen round the hand as being due to magnetic emanations from the body (using the word magnetic in a superphysical sense). They appear to think that the phenomenon is more rare than it actually is, and do not treat the case of cardboard hands. For the phenomenon as observed with these, there would seem to be two possible alternative explanations. One is, that the cardboard is occult cardboard, and the scissors hypermagnetic scissors, and that I have unwittingly impregnated everything with induced ectoplasmic activity. The other is that the phenomenon is a retinal (and rational) one, which can be observed whenever a white, or whitish, surface is seen in a feeble light, the visual purple from the actual retinal image diffusing into the neighbouring parts of the retina. Accepting, for argument's sake, the latter explanation (which accounts at once for the fact that nothing is seen in the dark), the effect will be intensified by the restless movement of the eye, which undoubtedly takes place when objects are viewed in unfavourable circumstances. ${ }^{1}$ The eye shifts the image into an unfatigued part of the retina, and the after image persists as a feeble glow. Such phenomena have been frequently described by Dr. EdridgeGreen in a variety of forms, and I do not claim any particular originality for this prosaic explanation.

But a further very interesting phenomenon can be observed with the cardboard hand, which has not, I believe, been described. If it be looked at fixedly, the ends of the fingers will be seen to vanish intermittently, now one, now the other, while the extended thumb and little finger appear to move up and down, producing somewhat the appearance of a hand opening and shutting. The effect is very striking, and is pleasantly diversified by the complete disappearance of the hand at intervals. This is due either to retinal fatigue, combined with eye movement, or else to the ferro-forcificatory magnetism of the scissors, permeated as they must be with psychic influences and what not. I must leave it to the readers of NATURE to repeat the experiments, and judge for themselves.

Seeing that the festive season (I understand that this is the correct way to refer to Christmas) is upon us, I venture to describe a third occult phenomenon, somewhat analogous to that quoted by Dr. EdridgeGreen in NaturE of December 9, p. 772. Two heads, facing one another, are cut out of white cardboard in profile, and observed in a very subdued light against a black background as before. (My heads are about two and a half inches in diameter, and the noses about half an inch apart.) By a delicate manipulation of the scissors one of the heads may be given a feminine character, largely by providing it with back hair. On careful observation the heads will be seen to approach and kiss repeatedly, separating with rapturous amaze after each contact. Like the other phenomena, including M. de Dubor's magnetic fluid, this cannot be observed in the dark, nor, I may add, even heard, in the case of the cardboard heads.

All the phenomena seem to be observed even more easily by myopic people than by myself. A morning's experiment has convinced me that with suitable illumination and white cardboard a very creditable séance can be arranged, including auræ, movements and levitations, magnetic emanations, and ectoplasm. This method involves no expense and no hymn singing. Even an atmosphere of reverence is not necessary for the production of the phenomena, although, I admit, the morning of my essay in the occult art was a Sunday morning, which may have had some favourable effect.

Artillery College, Woolwich,

December II.

\section{A Relativity Paradox.}

IT is with great diffidence that I enter the relativity controversy, since I know little or nothing of the subject. Ignorance, however, is seldom a bar to the expression of opinions. I understand that the fundamental idea underlying the theory of relativity is that no signal can be transmitted through space at a greater speed than the velocity of light. There appears to me, however, to be a method by which, in theory, it might be done, and since we have trains running past embankments with half the speed of light, and shells with observers inside travelling at even higher velocities, perhaps my observer at A (Fig. I) may be allowed to have two immensely long triangles made of any suitable material; A signals to B by sliding the two triangles together, one over the other, in the direction of the arrows; the point $X$, where the two sides intersect, moves towards the observer $B$, who receives the signal when he observes the point of intersection pass over him. If the angle at X is ro" and the triangles are moved together at a speed of ten miles a second (an absurdly small speed for a relativist), the signal will be transmitted to $B$ with more than twice the speed of light.

C. C.

Is not "C. C." assuming that when A shifts his triangles by tugging at their bases the apices instantaneously start to move? But the impulse would travel from base to apex at a speed far less than that of light, namely, the speed of elastic waves in the material. After the lapse of sufficient time the two triangles would move uniformly and as a whole; and the mechanism provides a good illustration of a recognisable point moving much faster than light. The relativist does not object to this, since the motion of $\mathrm{X}$ does not then correspond to anything coming within the definition of a signal. The time of signalling from $A$ to $B$ must be reckoned from the moment that $\mathrm{A}$ gives the impulse to the mechanism. A. S. Eddington.

1 See, e.g., Edridge-Green's "Physiology of Vision." (G. Bell and Sons.)

Observatory, Cambridge. 